\title{
Resolution enhancement at a large convergence angle by a delta corrector with a CFEG in a low-accelerating-voltage STEM
}

\author{
Hidetaka Sawada ${ }^{1}$, Takeo Sasaki ${ }^{1}$, Fumio Hosokawa ${ }^{1}$, and Kazutomo Suenaga ${ }^{2}$ \\ ${ }^{1}$ JEOL Ltd., 3-1-2 Musashino, Akishima, Tokyo, 196-8558, Japan \\ ${ }^{2}$ National Institute of Advanced Industrial Science and Technology (AIST), Tsukuba, 305-8565, Japan \\ hsawada@jeol.co.jp
}

\begin{abstract}
Resolution reduction by a diffraction limit becomes severe with an increase in the wavelength of an electron at a relatively low accelerating voltage. For maintaining atomic resolution at a low accelerating voltage, a larger convergence angle with aberration correction is required. The developed aberration corrector, which compensates for higher-order aberration, can expand the uniform phase angle. Sub-angstrom imaging of a Ge [112] specimen with a narrow energy spread obtained by a cold field emission gun at $60 \mathrm{kV}$ was performed using the aberration corrector. We achieved a resolution of $82 \mathrm{pm}$ for a Ge-Ge dumbbell structure image by high angle annular dark-field imaging.
\end{abstract}

Keywords: Ronchigram, higher-order aberration, corrector, STEM

\section{Introduction}

Observation at a low acceleration voltage is advantageous because of minimal damage to the specimens and a larger scattering cross-section resulting in a higher signal count for analysis in an electron microscope. The demand for an atomic-resolution instrument operating at a lower voltage is considerably increasing (Suenaga et al., 2009; Krivanek et al., 2009; Lee et al., 2012). However, it is difficult to perform high-resolution imaging because, at a low acceleration voltage, the longer wavelength of the electrons used for imaging increases the diffraction limit, so that the resolution worsens. The defocus spread $\left(d f_{\mathrm{Cc}}\right)$ due to chromatic aberration with an energy deviation of $d E$ is defined as $d f_{C c}=C c \cdot d E / U$, and a blur due to the chromatic aberration $d_{\mathrm{Cc}}$ is expressed as $d_{\mathrm{Cc}}$ $=C c \cdot d E / U \cdot \alpha$, where $U$ is the accelerating voltage and $\alpha$ is the convergence semi-angle. The blur causes a severe resolution reduction in a low-voltage microscope, because ratio $d E / U$ increases with a decrease in $U$, which in turn causes $d_{\mathrm{Cc}}$ to increase. For maintaining atomic resolution at a low accelerating voltage, a larger convergence angle with aberration correction and a narrow energy spread of the source to reduce the blur are required.

We have developed an electron microscope having higher-order aberration correctors, which are called delta correctors (Sawada et al., 2009a). The delta corrector system with three dodecapoles can compensate for a $3^{\text {rd }}$ order spherical aberration and a six-fold astigmatism. The developed microscope achieved high resolution at an accelerating voltage lower than $60 \mathrm{kV}$, by using a narrow energy spread obtained by a cold field emission gun (CFEG) (Kohno et al., 2010). The atomic-resolution imaging of a Si [110] specimen and a gold polycrystalline particle at $30 \mathrm{kV}$ and $60 \mathrm{kV}$ has been demonstrated by Sasaki et al. (2010). The probe sizes at the low accelerating voltage have been evaluated using the experimental data of a dark-field image obtained using a scanning transmission electron microscope (STEM) by comparing with the simulations (Sasaki et al., 2011). By using this instrument, the chemical information at atomic resolution is obtained by using electron energy loss spectroscopy (EELS) in the STEM (Suenaga et al., 2009; Suenaga et al., 2010; Suenaga et al., 2011).

In this article, sub-angstrom imaging using the developed microscope is demonstrated at a low acceleration voltage. To obtain a smaller probe to attain higher resolution, we optimized the convergence angle for STEM imaging and the Gaussian probe size for the specimen. For sub-angstrom-resolution imaging, we have used a germanium (Ge) crystalline specimen because the image in the [112] orientation shows a pair of two adjacent Ge atomic rows (dumbbell) separated by $82 \mathrm{pm}$, according to the geometrical configuration (O'Keefe et al., 2005). 


\section{Experimental setup}

An operating accelerating voltage of $60 \mathrm{kV}$ was used in the experimental test. We used a delta corrector equipped with a triple dodecapole and two sets of transfer lenses, and Ronchigrams were recorded for the evaluation of the spherical aberration corrector (Cs corrector) (Sawada et al., 2009a). To compare the aberration correction capability of the double-hexapole-type corrector and the delta corrector, Cs compensation using a double-hexapole system was tested. The contrast flat area of the Ronchigram reached 34 mrad with the correction using two hexapole fields, as shown in Fig. 1(a). The coherently converged angle was limited by the six-fold astigmatism $\left(A_{6}\right)$. Next, the delta corrector was used to correct the geometric aberrations, as shown in Fig. 1(b). For the delta optical system, the contrast flat area of the Ronchigram increased to $71 \mathrm{mrad} . \quad A_{6}$ was measured to be less than $0.05 \mathrm{~mm}$ from the experimental Ronchigram (Sawada et al., 2008). Figures 1(c) and 1(d) show the calculated phase with $A_{6}=2 \mathrm{~mm}$ and $0.05 \mathrm{~mm}$, respectively. The values of the $\pi / 4 \lambda$ limit, where the phase shift from the center becomes $\pi / 4$ because of aberration, are $32 \mathrm{mrad}(151 \mathrm{pm})^{-1}$ and $65 \mathrm{mrad}(75 \mathrm{pm})^{-1}$, respectively. With the delta corrector, a convergence angle of over 40 mrad is adaptable for high-resolution imaging using the STEM.

The microscope was equipped with a CFEG having a tungsten [310] tip to obtain a small energy spread of the source. The energy spread is varied by changing the extractor voltage. The full width at half maximum (FWHM) of the electron energy was measured at several settings, as shown in Fig. 2. In present observation, the energy spread of the source has an FWHM of $0.36 \mathrm{eV}$, after setting the probe current.
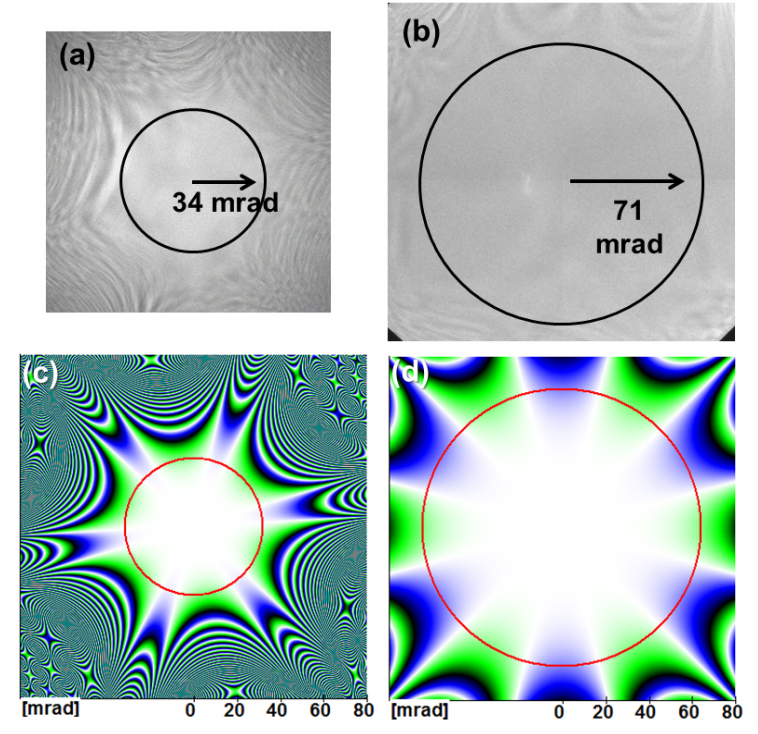

Fig. 1. Experimental Ronchigrams obtained using the (a) double-hexapole-type and (b) delta correctors at $60 \mathrm{kV}$. Calculated phase with (c) $A_{6}=2 \mathrm{~mm}$ and fifth-order spherical aberration $=1.5 \mathrm{~mm}$ and (d) $A_{6}=0.05 \mathrm{~mm}$ at $60 \mathrm{kV}$. White to black corresponds to $\pi$, and white to white corresponds to $2 \pi$. Circles describe the $\pi / 4$ limit.

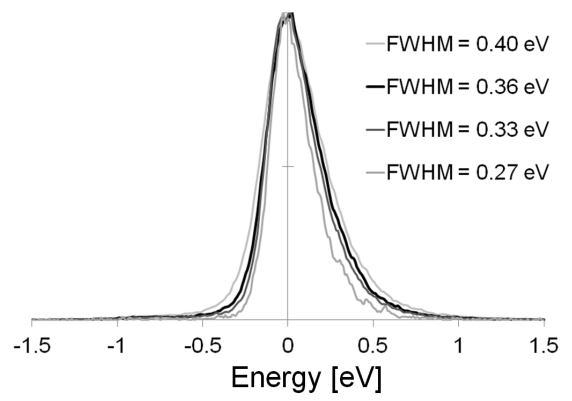

Fig. 2. Measured energy spreads of the CFEG at $60 \mathrm{kV}$ by varying the extractor voltage. Note that the exposure time $=0.1 \mathrm{~s}$, and dispersion $=0.01 \mathrm{eV} /$ channel using the Gatan Quantum ER with an entrance aperture of $1.5 \mathrm{~mm}$. 


\section{Simulations}

The probe size was calculated as a function of the convergence semi-angle at $60 \mathrm{kV}$. The simulation was based on the incoherent superposition method, using experimental optical parameters. The chromatic aberration was $0.84 \mathrm{~mm}$, and the energy spread of the CFEG was $0.36 \mathrm{eV}$ in this calculation. The Gaussian probe size of the specimen was set to be $14 \mathrm{pm}$. Here, D59 denotes the diameter of the electron beam that includes $59 \%$ of the total beam current (Haider et al., 2000). Notations D50, D75, and D90 are equivalent terms. The FWHM of the calculated probe monotonically decreases with an increase in the convergence semi-angle because of a smaller diffraction limit at a higher angle. On the other hand, D50, D59, D75, and D90 increase at angles over 40-50 mrad due to the blur caused by the chromatic aberration $d_{\mathrm{Cc}}=C c \cdot d E / U \cdot \alpha$, even though the probe-forming system has a small energy spread by CFEG and a small amount of a chromatic aberration coefficient. D50 has a minimum value at a semi-angle between $35 \mathrm{mrad}$ and $45 \mathrm{mrad}$, whereas the value of D59 is minimum at a semi-angle between $30 \mathrm{mrad}$ and $40 \mathrm{mrad}$. When a small D59 value is obtained, the signal-to-noise (S/N) ratio of the image is better because the tail of the probe is weak. On the other hand, a higher angle of over 40 mrad enables us to obtain sub-angstrom STEM imaging with a sharp-pointed probe.

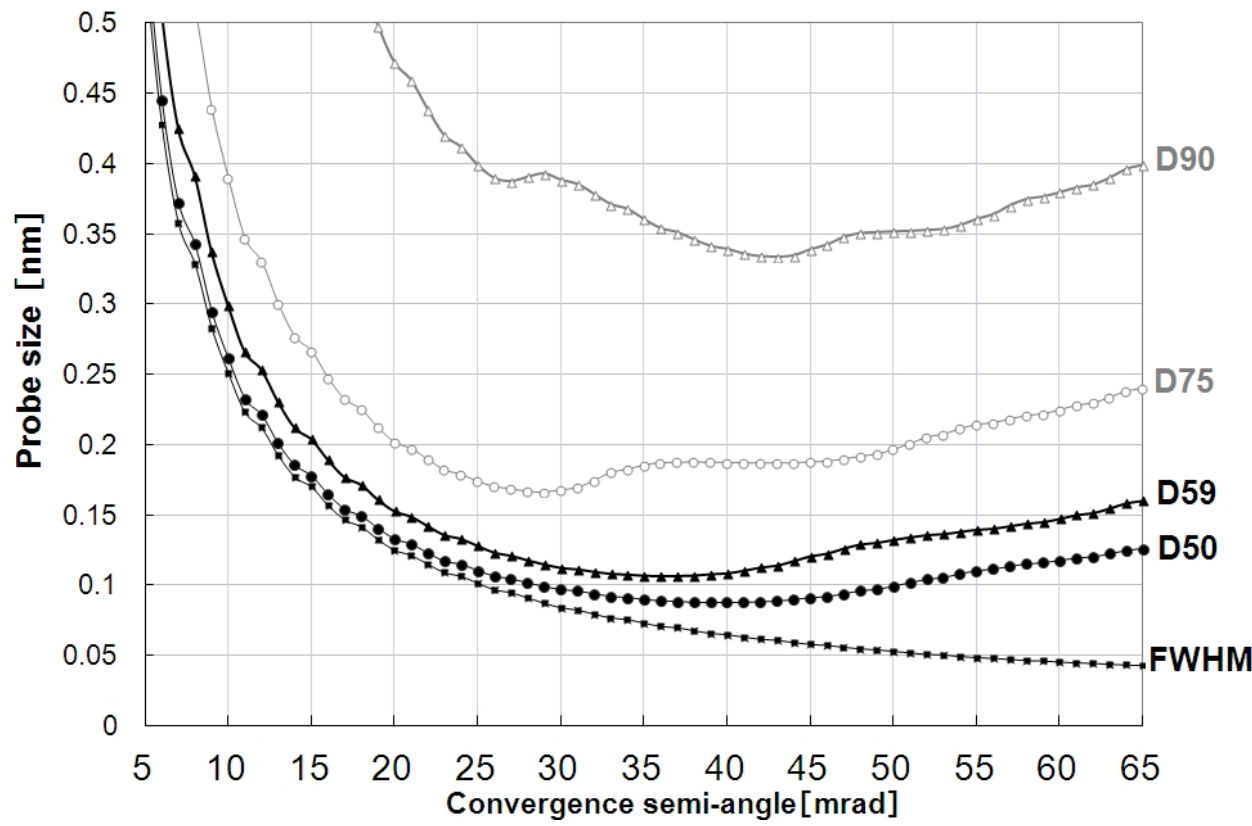

Fig. 3. Calculation of the probe sizes as a function of convergence semi-angle at $60 \mathrm{kV}$. Here, defocus $=0 \mathrm{~nm}$, $\mathrm{Cs}=0 \mathrm{~mm}$, and $A_{6}=0.05 \mathrm{~mm}$. Note that the probe was calculated with $4 \mathrm{pm} / \mathrm{pixel}$ in a real space size and 0.6 $\mathrm{mrad} /$ pixel on an aperture plane using a $2 \mathrm{k} \times 2 \mathrm{k}$ Fourier transform size. 
Figure 4(a) shows the calculated probe shape at convergence semi-angles of $30 \mathrm{mrad}, 35 \mathrm{mrad}, 40 \mathrm{mrad}$, and 45 mrad, and each probe size is listed in Table 1. The probe shape becomes sharper with an increase in the convergence semi-angle, resulting in a smaller FWHM. On the other hand, a larger convergence angle has a weaker probe tail. Thus, a larger convergence angle yields a smaller FWHM with slightly higher D50 and D59. Figure 4(b) shows the superimposition of the two simulated probes by shifting them $82 \mathrm{pm}$ apart, which corresponds to the distance between the atomic columns in a dumbbell seen in the Ge [112] crystal. While almost no dip was observed between the two peaks of the 30 -mrad probe, $19 \%, 41 \%$, and $57 \%$ dips were observed in the $35 \mathrm{mrad}, 40 \mathrm{mrad}$, and $45 \mathrm{mrad}$ probes, respectively. The probe simulation indicates that a $60-\mathrm{kV}$ probe at higher angle is achievable at the sub-angstrom scale by a smaller diffraction limit. Because the resolution of the high-resolution STEM image is influenced by not only the probe size but also the atomic potential, noise, etc., a sufficiently fine probe size is required to achieve the resolution of the dumbbells between two separate objects.
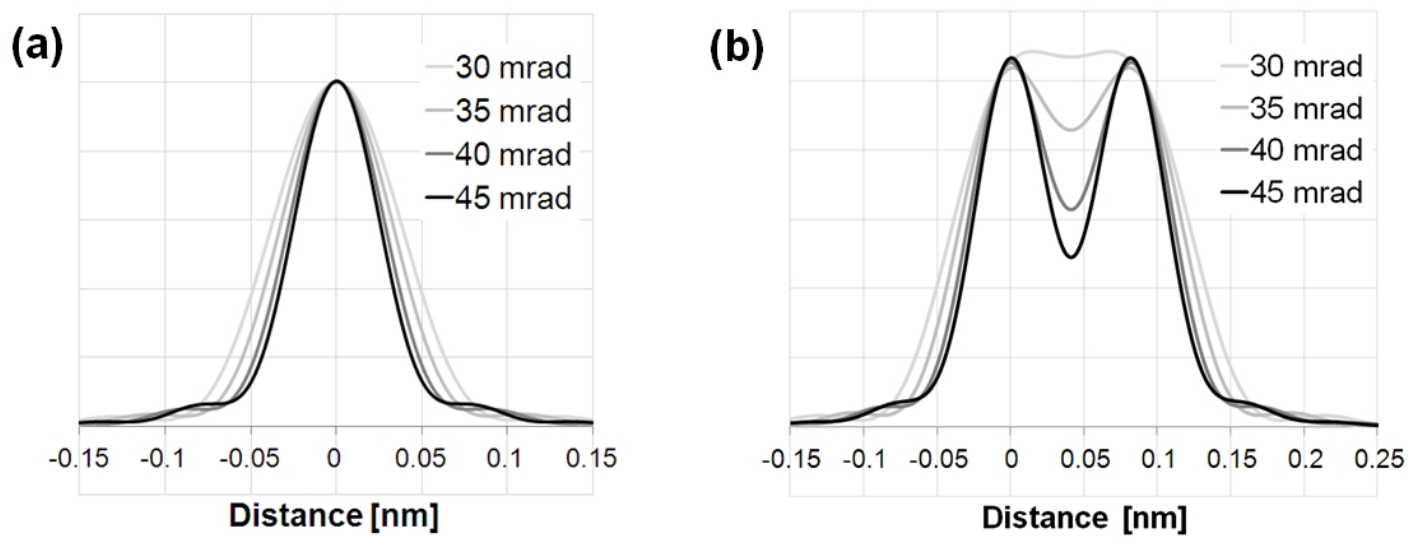

Fig. 4. (a) Calculated probe shapes at convergence semi-angles of $30 \mathrm{mrad}, 35 \mathrm{mrad}, 40 \mathrm{mrad}$, and $45 \mathrm{mrad}$. (b) Shapes of two superimposed and 82-pm shifted probes with distance of $82 \mathrm{pm}$ (Sawada et al., 2009b). Note that the probe was calculated with $2 \mathrm{pm} / \mathrm{pixel}$ in a real space size and $1.2 \mathrm{mrad} / \mathrm{pixel}$ on the aperture plane using a $2 \mathrm{k} \times$ $2 \mathrm{k}$ Fourier transform size. The chromatic aberration, energy spread, Gaussian probe size, defocus, Cs, and $A_{6}$ are the same as those used in the previous probe calculation.

Table 1

Calculated probe sizes

\begin{tabular}{|c|c|c|c|c|}
\hline & 30 mrad & 35 mrad & 40 mrad & 45 mrad \\
\hline FWHM [pm] & 83.9 & 73.0 & 64.8 & 57.8 \\
\hline D50 [pm] & 97.2 & 90.2 & 87.9 & 91.4 \\
\hline D59 [pm] & 112.1 & 106.6 & 108.3 & 120.4 \\
\hline D75 [pm] & 167.7 & 185.4 & 187.2 & 187.6 \\
\hline D90 [pm] & 388.4 & 360.3 & 339.2 & 338.9 \\
\hline
\end{tabular}


Figure 5 shows the simulated STEM images at $60 \mathrm{kV}$ at several convergence semi-angles. The dumbbell image was simulated by the multi-slice method by taking the dynamic and thermal diffuse scattering (TDS) into account (Hosokawa, 2013). The atomic dumbbell could not be clearly resolved at an angle less than 30 mrad. The large diffraction limit at a longer wavelength at a low accelerating voltage worsens the resolution when a relatively small distance is observed. At a convergence semi-angle of over $40 \mathrm{mrad}$, the 82 -pm dumbbell was resolved because of a smaller diffraction limit. The multi-slice simulation of the dark-field STEM images indicates that the 82-pm dumbbell can be resolved at a larger convergence semi-angle.
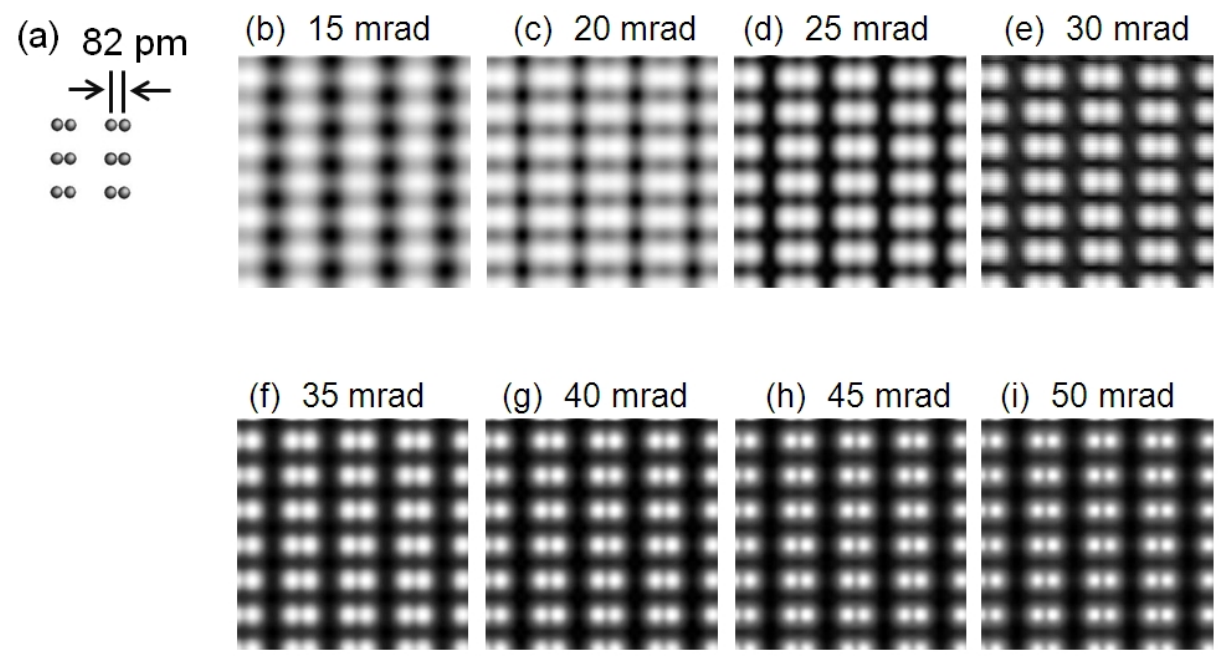

Fig. 5. (a) Structural model of Ge [112]. Simulated dark-field images of the Ge [112] specimen at convergence semi-angles of In the simulation the ADF-STEM images, a chromatic aberration of $0.84 \mathrm{~mm}$, an energy spread of 0.36, and a Gaussian probe size of $14 \mathrm{pm}$ were taken into account for all images by the convolution. All the axial aberration is set to zero. The detection angle for the dark field image is from 90 mrad to 200 mrad in the simulation. The thickness of the specimen used in this calculation is set to $10 \mathrm{~nm}$. (a) Structure model of Ge [112]. (b) The convergence semi-angles are (b) $15 \mathrm{mrad,} \mathrm{(c)} 20 \mathrm{mrad}$, (d) $25 \mathrm{mrad}$, (e) $30 \mathrm{mrad}$, (f) $35 \mathrm{mrad}$, (g) $40 \mathrm{mrad}$, (h) $45 \mathrm{mrad}$, and (i) 50 mrad at $60 \mathrm{kV}$ by performing a multi-slice method. 


\section{Results}

For evaluating the sub-angstrom resolution, a Ge [112] crystalline specimen was experimentally observed at 60 $\mathrm{kV}$, as shown in Fig. 6. The crystal orientation was adjusted with a double tilt specimen holder. In this observation, the energy spread of the source was measured to be $0.36 \mathrm{eV}$ for a total emission current of $5 \mu \mathrm{A}$. The convergence semi-angle was changed using round lenses between the condenser aperture and the STEM corrector. At $30 \mathrm{mrad}$, the Ge-Ge atomic column dumbbell was not resolved, although the $\mathrm{S} / \mathrm{N}$ of the image was better [Fig. 6(a)]. At the 40-mrad convergence semi-angle, shown in Fig. 6(c), the Ge-Ge dumbbells were resolved. Figure 6(d) shows that the 82-pm-separated dumbbell images are clearly seen at a convergence semi-angle of 45 mrad. Although the experimental images are not completely consistent with the simulated image shown in Fig. 5, they have a similar feature in that the dumbbell can be resolved at a higher angle.

Figure 7(a) shows a dark-field STEM image in the [112] orientation. The beam current was adjusted with an electrostatic lens around the CFEG. The probe has a current of $6 \mathrm{pA}$ and a convergent semi-angle of $45 \mathrm{mrad}$. The inner and outer detection angles of scattered electrons with the dark-field detector were 90 mrad and 200 mrad, respectively. The STEM image of an area of $5.9 \times 5.9 \mathrm{~nm}^{2}(512 \times 512$ pixels $)$ was recorded in $10 \mathrm{~s}$. An area of $3.6 \times 3.6 \mathrm{~nm}^{2}$ is shown in Fig. 7(a). Figure 7(b) shows the intensity profile of the dumbbell images aligned in the [1111] direction of the dotted region shown in Fig. 7(a). The intensity histogram is shown in Fig. 7(d). Figure 7(c) shows a Fourier transform of the STEM image shown in Fig. 7(a). The Ge-Ge dumbbells are resolved in Fig. 7(a), and the $44 \overline{4}$ spot (corresponding to $(82 \mathrm{pm})^{-1}$ ), the $\overline{6} \overline{2} 4$ spot (corresponding to $\left.(76 \mathrm{pm})^{-1}\right)$, and the $\overline{1} 7 \overline{3}$ spot (corresponding to $(74 \mathrm{pm})^{-1}$ ) are shown in Fig. 7(c). The 82-pm resolution was attainable at an accelerating voltage of $60 \mathrm{kV}$, where the electrons have a wavelength $(\lambda)$ of $4.866 \mathrm{pm}$.
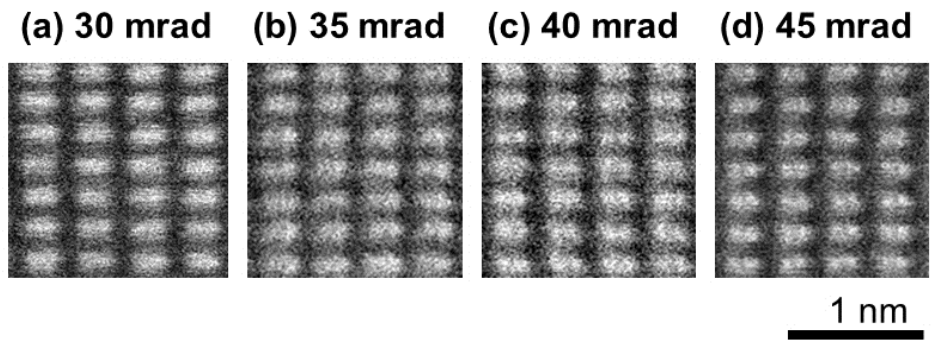

Fig. 6. Raw data of high-resolution dark-field images using a Ge [112] specimen for the (a) $30 \mathrm{mrad}$, (b) $35 \mathrm{mrad}$, (c) $40 \mathrm{mrad}$, and (d) $45 \mathrm{mrad}$ convergence semi-angles. 

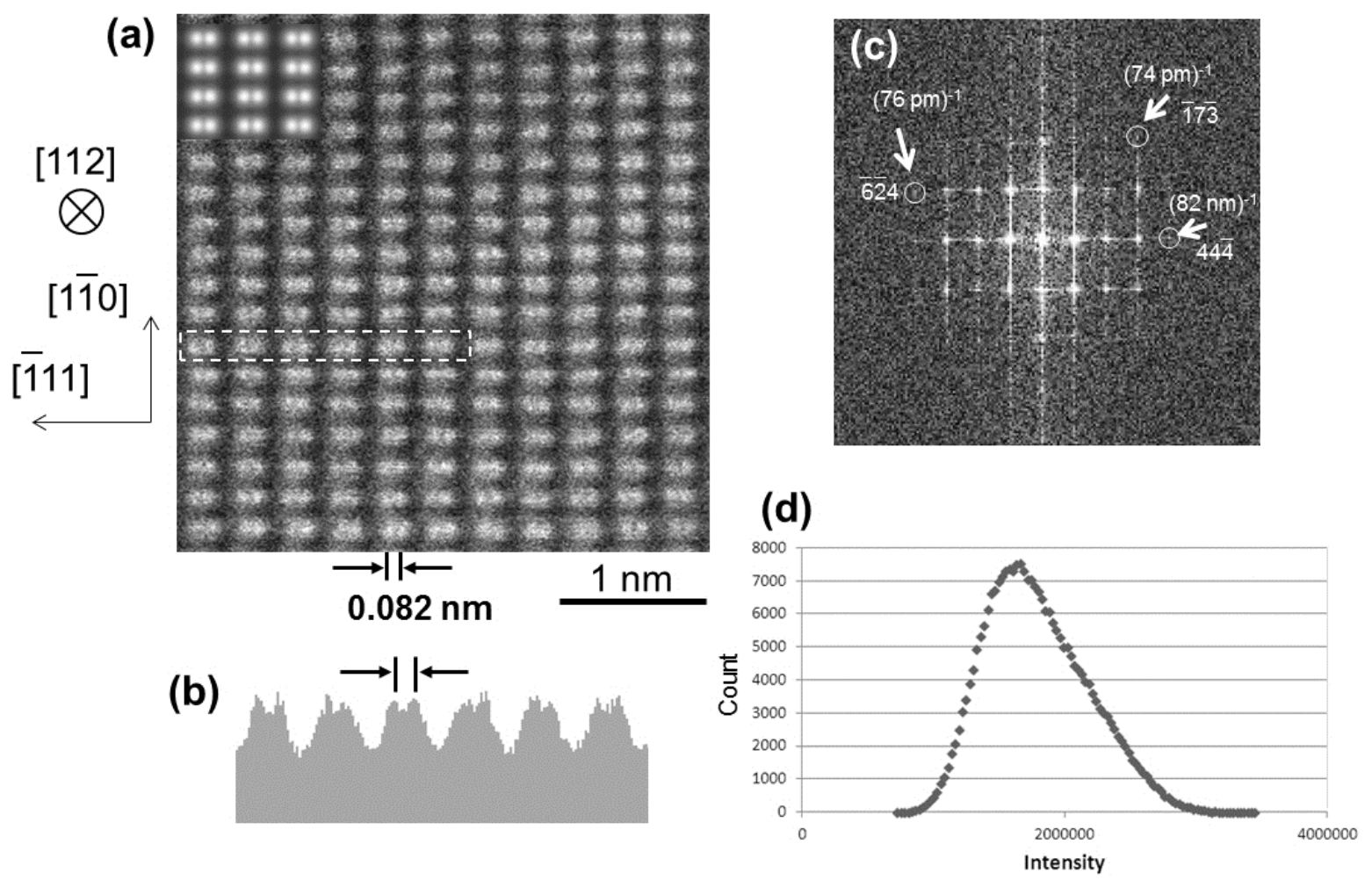

Fig. 7. (a) The Ge [112] dark-field image at $60 \mathrm{kV}$. Figure 7(a) shows a raw intensity image, where the incline of the vertical line is corrected by $1.5^{\circ}$ to align the dumbbells vertically to compare with the simulated image. The simulated image in Fig. 5(h) at 45 mrad is provided at the upper left corner of Fig. 7(a). The scanning speed is $38 \mu \mathrm{s} /$ pixel. (b) The intensity profile of the dotted region of Fig. 7(a) formed by averaging over a width of 8 pixels, showing the of Ge-Ge 82-pm dumbbells were resolved. (c) The power spectrum of the STEM image, showing spots of $(82 \mathrm{pm})^{-1}$. (d) The intensity histogram. 


\section{Conclusion}

The probe simulations indicated that, at a low accelerating voltage, high-resolution imaging at the sub-angstrom level required a relatively large convergence angle because of the longer wavelength of the electrons. By using a large uniform phase corrected by the delta corrector, we have observed the Ge-Ge atomic dumbbells of $82 \mathrm{pm}$ in a Ge [112] crystalline specimen to experimentally evaluate sub-angstrom-resolution imaging. The observation of the dark-field STEM imaging with the CFEG at an accelerating voltage of $60 \mathrm{kV}$ demonstrated the sub-angstrom resolution of the Ge-Ge 82-pm dumbbells with a Fourier spot of $(74 \mathrm{pm})^{-1}$. Resolution enhancement with a larger convergence angle and a narrow energy spread of the source was experimentally confirmed at a low accelerating voltage.

\section{Acknowledgments}

This work was supported by JST under the Research Acceleration Program (2012-2016).

\section{References}

Haider, M., Uhlemann, S., Zach, J., 2000. Upper limits for the residual aberrationsof a high-resolution aberration-corrected STEM. Ultramicroscopy 81,163-175.

Krivanek, O.L., Chisholm, M.F., Nicolosi, V., Pennycook, T.J., Corbin, G.J., Dellby, N., Murfitt, M.F., Own, C.S., Szilagyi, Z.S., Oxley, M.P., Pantelides, S.T., Pennycook, S.J., 2010. Atom-by-atom structural and chemical analysis by annular dark-field electron microscopy. Nature 464, 571-574.

Kohno, Y., Okunishi, E., Tomita, T., Ishikawa, I., Kaneyama, T., Ohkura, Y.,Kondo, Y., Isabell, T., 2010. Development of a cold field-emission gun for a $200 \mathrm{kV}$ atomic resolution electron microscope. Microsc. Anal. 24, S9-S13.

Lee Z., Meyer J. C., Rose H., Kaiser U., 2012. Optimum HRTEM image contrast at $20 \mathrm{kV}$ and 80 kV-Exemplified by grapheme. Ultramiscoscopy 112, 39-46.

O'Keefe M., Allard L., Blom D., 2005. HRTEM imaging of atoms at sub-Angstrom resolution. J. Electron Microsc. 54, 169-180.

Hosokawa, F, unpublished (2013).

Sasaki, T., Sawada, H., Hosokawa, F., Kohno, Y., Tomita, T., Kaneyama, T., Kondo, Y., Kimoto, K., Sato, Y., Suenaga, K., 2010. Performance of low-voltage STEM/TEM with delta corrector and cold field emission gun. J. Electron Microsc. 59, S7-S13.

Sasaki T., Sawada H., Okunishi E., Hosokawa F., Kaneyama T., Kondo Y., Kimoto K., Suenaga K., 2012. Evaluation of probe size in STEM imaging at 30 and $60 \mathrm{kV}$. Micron 43, 551-556.

Sawada H., Sannomiya T., Hosokawa F., Nakamichi T., Kaneyama T., Tomita T., Kondo Y., Tanaka T., Oshima Y., Tanishiro Y., Takayanagi K., 2008 Measurement method of aberration from Ronchigram by autocorrelation function. Ultramicroscopy 108, 1467-1475.

Sawada, H., Sasaki, T., Hosokawa, F., Yuasa, S., Terao, M., Kawazoe, M., Nakamichi, T., Kaneyama, T., Kondo, Y., Kimoto, K., Suenaga, K., 2009a. Correction of higher order geometrical aberration by triple 3-fold astigmatism field. J. Electron Microsc. 58, 341-347.

Sawada, H., Tanishiro, Y., Ohashi, N., Tomita, T., Hosokawa, F., Kaneyama, T., Kondo, Y., Takayanagi, K., $2009 b$. STEM imaging of 47-pm-separated atomic columns by a spherical aberration-corrected electron microscope with a 300-kV cold field emission gun. J. Electron Microsc. 58, 357-361.

Suenaga, K., Sato, Y., Liu, Z., Kataura, H., Okazaki, T., Kimoto, K., Sawada, H., Sasaki, T., Omoto, K., Tomita, T., Kaneyama, T., Kondo, Y., 2009. Visualizing and identifying single atoms using electron energy-loss spectroscopy with low accelerating voltage. Nat. Chem. 1, 415-418.

Suenaga, K., Koshino, M., 2010. Atom-by-atom spectroscopy at graphene edge. Nature 468, 1088-1090.

Suenaga K., Iizumi Y., and Okazaki T., 2011, Single atom spectroscopy with reduced delocalization effect using a 30 kV-STEM. Eur. Phys. J. Appl. Phys. 54, 33508-335011. 